\title{
Truthmaker Semantics for Relevant Logic
}

\section{Mark Jago ${ }^{1}$}

Received: 5 February 2019 / Accepted: 24 October 2019 / Published online: 7 January 2020

(C) The Author(s) 2020

\begin{abstract}
I develop and defend a truthmaker semantics for the relevant logic $\mathbf{R}$. The approach begins with a simple philosophical idea and develops it in various directions, so as to build a technically adequate relevant semantics. The central philosophical idea is that truths are true in virtue of specific states. Developing the idea formally results in a semantics on which truthmakers are relevant to what they make true. A very natural notion of conditionality is added, giving us relevant implication. I then investigate ways to add conjunction, disjunction, and negation; and I discuss how to justify contraposition and excluded middle within a truthmaker semantics.
\end{abstract}

Keywords Truthmaking · Truthmaker semantics · Relevant logic $\cdot$ Relevance Conditionality $\cdot$ Contraposition $\cdot$ Non-classical logic $\cdot$ Semantics

\section{Introduction}

This paper develops and defends a truthmaker semantics for relevant logic. Relevant logics developed first by way of proof theory $[5,19,20]$, followed by various formal semantics $[6,8,27-31]$, leaving open the issue of a suitable philosophical interpretation. Various attempts followed (e.g. [16, 18, 22]). (Restall [24] gives a good history of relevant logics.) My approach, by contrast, is to begin with a philosophical idea and develop it in various directions, so as to build a technically adequate relevant semantics.

The philosophical idea is based on truthmaking. We have states of affairs and a method of combining them together, and sentences are true or false in virtue of those states. This simple picture naturally harbours a persuasive notion of conditionality, which is neither classical or intuitionistic but relevant. So the question arises, can a

Mark Jago

mark.jago@nottingham.ac.uk

1 Department of Philosophy, University of Nottingham, University Park, Nottingham NG7 2RD, UK 
truthmaker semantics be developed for a whole system of relevant logic? I argue that it can. I focus on the most well-known relevant logic, $\mathbf{R}$ (set out in Appendix A). My strategy is to argue, on philosophical grounds, for a number of principles governing truthmaker semantics and to show that, taken together, these give a sound and complete formal semantics for $\mathbf{R}$.

My approach first adopts a notion of conditionality (Section 3), to which I then add conjunction (Section 4), disjunction (Section 5), and negation (Section 6). I then discuss how to accommodate the stronger principles of contraposition (Section 7) and excluded middle (Section 8). The technical appendices set out $\mathbf{R}$ (Appendix A), the formal semantics and its key properties (Appendix B), and proofs of soundness and completeness (Appendix C). Throughout, I shall focus on developing the truthmaker approach and discussing the philosophical issues it raises, rather than discussing alternative semantics or their philosophical interpretations.

By way of further motivating the approach, I begin (Section 2) with a puzzle, relating relevance, conditionality, and validity. I then argue (Section 3) that the truthmaker approach provides a very elegant solution.

\section{The Problem of Relevance}

Relevant logics seek to avoid 'fallacies of relevance', or 'paradoxes' of the material or strict conditional, such as:

(1) $A \rightarrow(B \rightarrow B)$

(2) $A \rightarrow(B \vee \neg B)$

(3) $(A \wedge \neg A) \rightarrow B$

These are not valid in relevant logics. But $B \rightarrow B$ is valid in all relevant logics, and $B \vee \neg B$ is valid in strong ones. So to avoid (1) and (2), the semantics requires points of evaluation where $B \rightarrow B$ and $B \vee \neg B$ fail to obtain. Yet if these can fail to obtain, what entitles a relevant logician to call them valid? So a first problem for a relevant semantics is this:

Problem 1: How can $A \rightarrow A$ (and $A \vee \neg A$ ) fail to obtain and yet still be valid?

Taken as a purely technical problem, a solution is easy to find. We can distinguish normal from non-normal points, allow $A \rightarrow A$ and $A \vee \neg A$ to fail at non-normal points only, and define validity as whatever obtains at all normal points in all models. But the problem I have in mind is philosophical, concerning our concept of validity, and not merely a technical one:

PROBLEM 2: How can a semantics which solves PROBLEM 1 be philosophically justified?

If a semantic point of evaluation is good for counterexampling an inference or a conditional, why is it not good for counterexampling a claim to validity? It seems we must simply stipulate that some points are to count for purposes of validity and some are not. That doesn't answer our question; it merely stipulates that it's not to be asked.

The solution I offer is in terms of truthmakers, the entities in virtue of which truths are true. 


\section{The Conditional}

Truths are true in virtue of certain situations or states of affairs obtaining. (I'll speak of states.) To say that a truth is true in virtue of a specific state is not merely to say that the state guarantees that truth. On the intended sense, 'Socrates drank hemlock' is true in virtue of the specific state that Socrates drank Hemlock but not in virtue of the entire state of classical Athens, even though that big state clearly suffices for that truth. A truthmaker for a truth should be just enough - neither too much nor too little - to make the truth true [25]. Fine [11-13] calls this exact truthmaking, whereby a truthmaker 'must be relevant as a whole to the truth of the statement' [11, 628]. It is this exact notion of truthmaking which will be operative throughout.

We might then understand the implication from $A$ to $B$, in broad outline, as follows. Beginning with some state, suppose we were to add to it any truthmaker for $A$. Would we then arrive at a state in virtue of which $B$ is true? If so, then the original state counts as a truthmaker for the conditional $A \rightarrow B$. This is just the beginnings of an analysis of the conditional. We will need to understand what 'adding' states amounts to. But note already an important feature of the approach: enlarging a state in virtue of which $A$ is true need not result in a state in virtue of which $A$ remains true. We can expand the specific state in which Socrates drinks hemlock to encompass the state of Athens, which (as noted above) is not a truthmaker for 'Socrates drank hemlock'. So, on our analysis, the state Athens is not a truthmaker for:

(4) If Socrates drinks hemlock then Socrates drinks hemlock

Likewise, the states that I am currently typing and that wombats waddle are not truthmakers for (4). Very few states are. Note how these states are perfectly normal, with no special metaphysical or logical quirks. We thus have a very natural explanation of how instances of $A \rightarrow A$ may fail at a state. We get this because our evaluative relation between state and sentence holds when that sentence is true in virtue of (and not merely relative to) that state.

What then of validity? A valid sentence is one that is true whatever, irrespective of which states of affairs obtain. Given any states of affairs, or none, a valid sentence will be true. Its truth depends on nothing in particular; its demand on reality is nothing at all. It is not that valid sentences lack truthmakers. 'I am typing or not typing' is true because I'm typing; and, on our analysis, that Socrates drank hemlock is a truthmaker for (4). The point is that (4) would be true even if Socrates hadn't drank hemlock. Its truth demands nothing of the world.

It is useful to have a way of evaluating sentences with respect to nothing (with apologies to Lewis Carroll). Just as the empty set is a collection with no members, the null state is a state that is, or represents, nothing at all. If we accept this notion as an abstraction, or what Forrest $[15,79]$ call as 'fictitious item', or some other way - then we have a very intuitive truthmaking analysis of validity as being made true by the null state. This criterion immediately pays dividends. Adding a truthmaker for $A$ to the null state results in none other than that same truthmaker for $A$. So, on our analysis, the null state is guaranteed to make $A \rightarrow A$ true. $A \rightarrow A$ is therefore valid, even though we have states available which do not make $A \rightarrow A$ true: exactly the desired result. (I'll return to the validity of $A \vee \neg A$ in Section 8.) 
None of this would get us very far if we could not pin down some reasonable notion of 'putting states together'. Luckily, we may appeal to ideas that have become standard in truthmaker semantics. At its core, this is just the idea sketched above on which sentences are evaluated relative to states, with the evaluation relation, ' $\vdash$ ', understood in terms of the sentence's being true in virtue of that state. Truthmaker semantics comes with a built-in notion of 'putting two states together'. All states in the domain are ordered by a partial order (reflexive, transitive, and anti-symmetric) $\sqsubseteq$, with a minimal element $\square$ (the null state), such that any two states $s, u$ have a least upper bound, $s \sqcup u$ in the domain. This ordering $\sqsubseteq$ is typically interpreted as the parthood relation, with ' $s \sqsubseteq u$ ' read as: $s$ is a part of $u$. Under this interpretation, ' $s \sqcup u$ ' denotes the mereological sum (or fusion, or composition) of $s$ and $u$. So we can interpret talk of 'adding state $u$ to state $s$ ' as resulting in the state $s \sqcup u$.

Truthmaker semantics is a natural framework within which to locate a semantics for relevant logics, for at least three reasons. First, invoking the 'true in virtue of' locution requires that truthmakers be relevant to what they make true. Not every state makes ' $a$ is either red or not red' true. That $a$ is red does, but that $b$ is blue does not. The notion of relevance here is left at the intuitive level, just as it is in relevance logic; but still, there does seem to be a confluence of motivation. Second, as just noted, truthmaker semantics pins down a suitable notion of 'putting two states together' which (as we shall see below) gives rise to a suitable conditional. And third, as we just saw, it provides an intuitive notion of validity (via the null state, a stock character of truthmaker semantics) on which $A \rightarrow A$ is valid.

With these minimal resources, we can begin setting out the semantics for the conditional (with the other connectives to appear through Sections 4-6). A truthmaker $s$ for $A \rightarrow B$ is a state which, whenever combined with a truthmaker for $A$, results in a truthmaker for $B$ :

$(\rightarrow) s \Vdash A \rightarrow B$ iff, for all states $u$ such that $u \Vdash A, s \sqcup u \Vdash B$.

Urquhart $[30,31]$ gives a technically identical clause, but without the truthmaking interpretation (he speaks of 'pieces of information').

Not only is $(\rightarrow)$ very natural within truthmaker semantics, it also gives exactly the results we're after. As Urquhart shows, to obtain the conditional of $\mathbf{R}$ we require $\sqcup$ to be idempotent $(s \sqcup s=s)$, commutative $(s \sqcup u=u \sqcup s)$, and associative $((s \sqcup t) \sqcup u=s \sqcup(t \sqcup u))$. These are mathematically guaranteed in truthmaker semantics by our definition of $\sqcup$ in terms of the partial order $\sqsubseteq$ and they are philosophically justified on our interpretation of $\sqsubseteq$ as parthood. (By contrast, there's no guarantee that 'pieces of information' will behave like this. Order and repetition may be essential to some instructions, for example.)

So adding $(\rightarrow)$ to the semantics results in a conditional which behaves just like the relevant conditional (at least, when no other connectives are around). Now let's see what happens when we consider more connectives, beginning with conjunction.

\section{Conjunction}

Adding conjunction to our account is straightforward from a purely logical point of view, but it raises philosophical questions of interpretation. Classically and 
intuitionistically, conjunction is that connective which licences the inference from a pair of sentences to their combination and back again, and which allows us to move between premises and conditional antecedents.

In relevant logics, these roles split into two. Extensional conjunction, written ' $\wedge$ ', is governed by the axioms $A \wedge B \rightarrow A, A \wedge B \rightarrow B$ and the adjunction rule: from $A, B$, infer $A \wedge B$. But it does not allow us to transform conjoined premises into conditional antecedents. If it did, we could move from $A \wedge B \vdash A$ (valid in any relevant logic) to $B \vdash A \rightarrow A$ (which is one of those irrelevant inferences which relevant logics seek to avoid).

Intensional conjunction, ' $\circ$ ', by contrast, preserves the inferential connection between premises and conditional antecedents, known as residuation:

$$
A \circ B \vdash_{\mathbf{R}} C \text { iff } A \vdash_{\mathbf{R}} B \rightarrow C
$$

The trade-off is that $A \circ B$ does not imply either conjunct on its own. So neither $\wedge$ nor $\circ$ preserve all features of 'and' to which classical and intuitionistic logic have accustomed us.

Given that $A \wedge B \rightarrow A$ is valid, the clause for $\wedge$ has to be the usual-looking one: $(\wedge) \quad s \Vdash A \wedge B$ iff $s \Vdash A$ and $s \Vdash B$.

The clause for $\circ$ likewise is already fixed. If adding a $B$-state to an $A$-state always results in a $C$-state then, residuation tells us, any $A \circ B$-state is a $C$ state. Since this holds for any $C$, the $A \circ B$-states must be the sum of an $A$-state with a $B$-state:

(o) $s \Vdash A \circ B$ iff there are states $s, u$ such that $s=t \sqcup u, t \Vdash A$, and $u \Vdash B$.

(The permutation axiom of $\mathbf{R}$ implies $A \circ B \vdash_{\mathbf{R}} B \circ A$, which concurs with our symmetrical treatment of summation.) Given these clauses, we have a sound and complete semantics for the $\rightarrow / \wedge /$ o fragment of $\mathbf{R}$ (Appendix $C$ ).

How should we understand $\circ$ and $\wedge$, as defined in relevant logic, given that neither preserves all the inferential features a competent user expects of 'and'? In discussing the issue, [17] offers an explanation of the two conjunctions in terms of situations carrying information. A situation $s$ carries the information that $A \wedge B$ when it carries the information that $A$ and the information that $B$. By contrast, $s$ carries the information that $A \circ B$ by carrying the information that there are other states, $s_{1}$ and $s_{2}$ 'in the same world', which carry the information that $A$ and that $B$, respectively $[17,9]$. But this understanding is problematic, if we understand "carries the information that $A$ ' to be factive. For if $s$ carries the information that some other existent state carries the information that $A$, then $s$ carries the information that $A$, and so $A \circ B$ collapses into $A \wedge B$.

More generally, relevant logicians assume that the inferential patterns we intuitively associate with 'and' split in relevant logic between $\wedge$ and $\circ$. On that view, we are wrong to treat 'and' as a unified concept governed by these inferential patterns. There must be two 'and'-concepts in common usage, a fact hidden to introspection and conceptual reflection but uncovered relatively recently by relevant logicians. That is deeply implausible. Truthmaker semantics, I suggest, can offer a better explanation.

Truthmaker semantics is committed to reading ' $\circ$ ', rather than ' $\wedge$ ', as English 'and'. For truthmaker semantics makes use of the exact, 'in virtue of' concept of truthmaking (Section 3), on which ' $A$ and $B$ ' is true in virtue of the state consisting of a truthmaker $A$ plus a truthmaker for $B$ [25]. This clause, first suggested by [32], 
is characteristic of exact semantics; and it is precisely the clause for (o). In giving an exact semantics, therefore, we are committed to aligning the connective defined by (o) with the concept we express in English using 'and'.

How can I insist that ' $O$ ' be read as 'and', when ' $A$ and $B$ ' clearly implies ' $A$ ' whilst ' $A \circ B$ ' does not? Let me explain. Truthmaker semantics discerns an exact and an inexact notion of truthmaking, where an inexact truthmaker for $A$ is any state which has an exact truthmaker for $A$ as a part. These give rise to exact and inexact notions of inference. Both can be seen as good forms of reasoning, each on their own terms. The former preserves specific grounds for truth, and hence relevance; the latter preserves just the existence of a ground for truth, which need not maintain relevance. Of course conjunctions entail their conjuncts, in the truth-preservation sense. And indeed, on the inexact sense, $A \circ B$ entails $A$. On this explanation, it is the concept of good reasoning that splits, not the concept of conjunction. So $\circ$ is maintained as the univocal concept of conjunction, with different inferential behaviours depending on which notion of reasoning (exact or inexact) is in play.

Can't a parallel argument by run in favour of treating $\wedge$ as the univocal notion of conjunction? No. The argument takes standard truthmaker semantics as its starting point, on which the treatment of 'and' is clear: it is that connective which satisfies the semantic clause (o) in the exact setting. The explanation is available only if we adopt the philosophical perspective on which o (as we notate it here) is genuine 'and'.

What then of $\wedge$ ? Its exact semantics suggests an 'overlapping content' interpretation: ' $A \wedge B$ ' says that $A$ and $B$ have a truthmaker in common. That state is an exact truthmaker for $A \wedge B$, just as $(\wedge)$ says. We may be interested in whether some mental statement $A$ has a physical realiser, for example. This will be the case if $A$ and the disjunction $P$ of all physical statements have a common truthmaker [14], that is, if $A \wedge P$ (as understood here) is true. Note that a state may inexactly truthmake both $A$ and $B$ without their having an exact truthmaker in common. As a result, we cannot reason inexactly from $A, B$ to $A \wedge B$. Unlike $\circ, \wedge$ does not coincide with classical conjunction within inexact reasoning.

\section{Disjunction}

When we turn to disjunction, the semantic picture becomes more complex. Interpreting disjunction in the usual extensional way:

( $\vee$ E) $s \Vdash A \vee B$ iff $s \Vdash A$ or $s \Vdash B$

results in a logic strictly stronger than $\mathbf{R}^{+}$, the negation-free fragment of $\mathbf{R}$ (Urquhart [30]; see Fine [9] for further results). There are independent reasons to be wary of $(\vee E)$ within an exact semantics. The state of my having a (binary) sibling makes it true that I have either a sister or a brother, but it doesn't make it true that I have a brother and it doesn't make it true that I have a sister. We should not identify the former 'disjunctive' state of affairs with either of the more determinate 'disjunct' ones, for they have different identity and persistence conditions. They involve different properties and, were my brother to change his gender to female, the sibling state would remain but the brother state would not. 
I suggest that states may stand to one another in a non-causal relation of determination or refinement. That I have a brother determines that I have a sibling. That the box is scarlet determines that the box is red. In each case, the former refines the latter, making it more precise. The notion I have in mind is related to the traditional determinatedeterminable classification [21, 33]. But (as I intend the notion and unlike on the traditional one) distinct determinates need not exclude one another: I may have both a sister and a brother, with each state individually determining that I have a sibling. I'll use 'determines' and 'is determined by' in this sense throughout, writing ' $s \prec u$ ' for ' $s$ determines $u$ ' and (as will be more common) using ' $\preceq$ ' for the non-strict analogue, a partial order on states. We need not worry whether there exist absolutely determinate states, for certain states are determinate enough for our purposes: those (relative to a fixed language) which make true a disjunct of each disjunction they make true. These are the prime states (which may or may not be atoms of $\preceq$ ). We will work with a set $P$ of prime states and write $s \preceq_{P} u$ when $s$ is a prime determinate of $u$.

With this in mind, an analysis of disjunction presents itself. The state that I have a (binary) sibling makes it true that I have either a brother or a sister because any prime determinate of it is a truthmaker either for 'I have a brother' or for 'I have a sister'. Similarly, the state of affairs that the box is red makes true the disjunction 'the box is scarlet or crimson or ...' because, however we make it determinate enough, we get a truthmaker for 'the box is scarlet' or for 'the box is crimson' or .... So our clause for disjunction is as follows:

( $) s \Vdash A \vee B$ iff, for all $u \preceq P s, u \Vdash A$ or $u \Vdash B$.

Our partial orders $\sqsubseteq$ and $\preceq$ should interact in various ways. Since that $a$ is scarlet determines that $a$ is red, we should also say that $a$ is scarlet and $b$ is square determines that $a$ is red and $b$ is square. Generalising:

(C2) If $s \preceq u$ then, for any $t, s \sqcup t \preceq u \sqcup t$.

Given this principle, that the box is scarlet and I have a brother determines both that the box is red and I have a sibling $(t \sqcup u)$ and that the box is scarlet and I have a sibling $\left(t^{\prime} \sqcup u\right)$, in which a part of the latter determines the corresponding part of the former. Our second principle generalises this relationship for prime determinates:

(C3) If $s \preceq_{P} t \sqcup u$, then there is a $t^{\prime} \preceq_{P} t$ such that $s \preceq_{P} t^{\prime} \sqcup u$.

Given the examples, these principles seem well justified. The resulting semantics is sound and complete for the positive fragment $\mathbf{R}^{+}$(Appendix C).

Taking stock: truthmaker semantics, augmented with the notion of determination and a natural notion of conditionality, results in an intuitive, natural, and philosophically justified semantics for $\mathbf{R}^{+}$. Now we turn to negation, where the going will be harder.

\section{Negation and False Conditionals}

Truthmaker semantics comes with a built-in approach to negation, through the concept of falsitymaking. States make propositions false, just as they make other propositions true. The state that I'm sitting makes it false that I'm standing up, false that no one is sitting, and false that I'm an immaterial entity. A truthmaker for a negated proposition $\neg A$ is then whatever would make $A$ false; and similarly, a falsitymaker for $\neg A$ is whatever would make $A$ true. We then require falsitymaking clauses for 
the other connectives, in parallel to their truthmaking clauses. Given the De Morgan equivalences, the truthmaking cases for $\wedge$ and $\vee$ must follow the truthmaking clauses for $\vee$ and $\wedge$, respectively; and given residuation, a falsitymaker for $A \circ B$ must be any state which, when combined with a truthmaker for one conjunct, results in a falsemaker for the other. (The clauses are listed in full in Appendix B, definition 2.)

The one piece still missing is an account of how the conditional is made false. A falsitymaker for a conditional $A \rightarrow B$ should (in some sense) be a counterexample to it. But we cannot take this to mean a state whose sum with a truthmaker for $A$ is not thereby a truthmaker for $B$. For then, $s$ would make false any conditional it does not make true, so that any state would make $(A \rightarrow B) \vee \neg(A \rightarrow B)$ true and hence the irrelevant $C \rightarrow((A \rightarrow B) \vee \neg(A \rightarrow B))$ would be valid. An alternative is to consider a further way of combining states, $\bar{\square}$, under which the combination of a conditional falsitymaker and an antecedent truthmaker will fail to make the consequent true. But this approach, whilst technically adequate, is unappealing in the present setting. The appeal of $(\rightarrow)$ is that it uses the intuitive and philosophically well-understood part-whole relation. Nothing similar can be said for $(\bar{\square})$.

A better approach is to consider compatibility relations that hold between states [4, 23]. Intuitively, states $s$ and $u$ are compatible when what one makes true the other doesz not make false and vice versa. But for the purposes of our semantics, we want to take compatibility as a primitive concept and constrain the truthmaking and falsitymaking relations accordingly. Since compatibility is intuitively well understood, it seems reasonable to take it as a primitive (just as many theories of modality take the possibility of a world as a primitive notion). To obtain a full semantics for $\mathbf{R}$, we will need to make sense of one state $s$ 's being maximally compatible with another, $u$, such that if one doesn't truthmake $A$, the other makes it false. Such states form an exhaustive pair.

I'll discuss below whether this assumption is reasonable. For now, let's see where it gets us. First, note that both compatibility and exhaustiveness are symmetrical but non-reflexive. A state will not be compatible with itself when it makes some $A$ both true and false; and it will form an exhaustive pair with itself just in case it itself makes each $A$ either true or false. Following [26], let's say that each state in an exhaustive pair is the mate of the other. We may think of matehood as a function from states to states, such that $s$ 's mate's mate is $s$ itself. Writing $s^{M}$ for the $s$ 's mate, the conceptual picture we have is that:

$$
s \Vdash A \text { iff } s^{M} \text { Al } A \quad s \dashv A \text { iff } s^{M} \Vdash A \quad s^{M M}=s
$$

We arrived at this picture by considering the concepts of compatibility and exhaustiveness. However, these shall play no official role in the formal semantics. Rather, we shall assume a primitive matehood function, defined so that $s^{M M}=s$ and constraining valuations so that the biconditionals above hold for primitive sentences. (We then show that the biconditionals hold for arbitrary sentences, via the crossover lemma 3 .)

Armed with the notion of a mate, we can now return to the issue of falsitymaking for conditionals. As a first approximation, we take a state to be a falsitymaker for a conditional when its mate is a counterexample:

(5) $s \dashv A \rightarrow B$ iff $\exists u \Vdash A: s^{M} \sqcup u \Vdash B$

An issue we must address is the interplay of matehood and non-prime states. A non-prime state $s$ makes true some disjunction $A \vee B$, but neither $A$ nor $B$. If it had a 
mate $s^{M}$, it would make both $A$ and $B$ and hence $A \vee B$ false. But then $s^{M}$ cannot be $s$ 's mate, since it makes false something $s$ makes true. So we must restrict the concept of matehood to prime states. This is no problem, for the truthmaking and falsitymaking behaviour of non-prime states is fully captured by their prime determinates. A non-prime state $s$ truthmakes (and falsitymakes) whatever all its prime determinates truthmake (and falsitymake):

(Heredity) $s \Vdash A$ iff $u \Vdash A$ for all $u \leq_{P} s$; and $s \dashv A$ iff $u \dashv A$ for all $u \leq_{P} s$.

(See lemma 2, Appendix B.) So absent a mate for non-prime $s$, we can work with the mates of $s$ 's prime determinates. In particular, we can take non-prime $s$ to be a falsitymaker for a conditional $A \rightarrow B$ when all its prime determinates satisfy (5):

$(\rightarrow \mathrm{F}) s \dashv A \rightarrow B$ iff $\forall t \leq_{P} s \exists u \Vdash A: t^{M} \sqcup u \Vdash B$

When $s$ is a prime state, this implies (5) (corollary 1b) and so we may adopt $(\rightarrow \mathrm{F})$ in general, whether $s$ is prime or not.

We could now revisit our analysis of the other connectives, using matehood to give each a falsitymaking clause. In fact, we could avoid a falsitymaking clause altogether and give a clause for negated sentences directly in terms of matehood (corollary 2), as is standard in relevant semantics (where the Routley star is used in place of matehood). This however leads to a definition of negation in terms of incompatibility, which risks conceptual circularity. ((Berto and Restall [4] argue otherwise). It is preferable within truthmaker semantics to analyse negation in terms of falsitymaking, with matehood acting as a restriction on (and not a definition of) truthmaker-falsitymaker pairs.

This approach assumes all prime states have a mate. Is that assumption reasonable? Not if we align states with Armstrong's states of affairs, with each characterised in terms of a particular having a property, or some particulars standing in some relation [3]. The state of affairs that Anna is wistful involves Anna herself and the property of being wistful, but there is no Armstrong-style state which makes false all that this does not make true: that Bec is creative, that Cath is rich, and so on.

For semantic purposes, we should not tie the notion of a state to a particular metaphysical view. To make sense of a sensible notion of entailment, we need enough freedom to provide a suitable stock of counter-models. This requires a valuation function (fixing truthmaking and falsitymaking for atomic sentences) which is relatively unconstrained, restricted only by the structural requirements imposed by determination and matehood (conditions (C1) and (C1) in Appendix B). Given this approach, the existence of mates is unproblematic.

\section{Contraposition}

How should matehood interact with summation and determination? The interaction will affect how negation behaves within a conditional, as in the case of conditionals which contrapose and support reductio (both of which are valid in $\mathbf{R}$ ):

(Contraposition) $(A \rightarrow B) \rightarrow(\neg B \rightarrow \neg A)$

(REductio) $(A \rightarrow \neg A) \rightarrow \neg A$

These axioms imply corresponding rules, preserving validity from antecedent to consequent. It is hard to deny that a conditional should obey the contraposition rule. And 
indeed, it holds in the truthmaker semantics with mates, without any conditions relating matehood to summation or determination (corollary 6, Appendix B). The fact that we get (rule) contraposition for free is a further indication that truthmaker models are a natural home for the conditional.

We cannot infer from the contraposition rule to the axiom, however. The axiom is stronger: it requires every state which makes $A \rightarrow B$ true to also make $\neg B \rightarrow \neg A$ true. (The rule requires this only of the null state.) This won't be the case in general, as the following model shows (with lines indicating parthood):

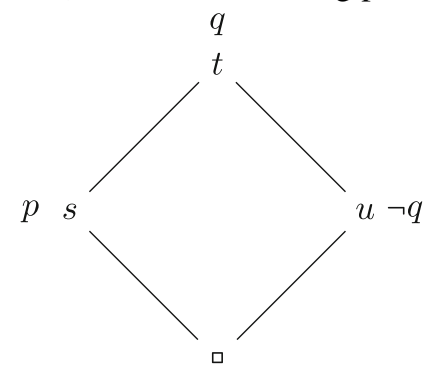

Here, $u$ truthmakes $p \rightarrow q$, since the only $p$-state is $s$ and their sum $t(=s \sqcup u$ ) truthmakes $q$. But $u$ doesn't truthmake $\neg q \rightarrow \neg p$, since it falsemakes $q$ and yet, summed with itself $(u \sqcup u=u$ ), it doesn't falsemake $p$. (That valid rules do not always translate into valid conditionals is a quirk of relevant logic, but not just of relevant logic. Necessitation (from A's validity to $\square A$ 's) is valid in normal modal logics, but $A \rightarrow \square A$ is certainly not.)

Validating CONTRAPOSITION requires interaction between matehood, summation, and determination so that, if $s$ and $u$ are prime and $s$ determines $u$ 's sum with some other state $t$, then $u^{M}$ determines $s^{M}$ 's sum with $t$ :

(C1) If $s \preceq t \sqcup u$ then $u^{M} \preceq t \sqcup s^{M}$

If we accept $(\mathrm{C} 1)$, then the resulting truthmaker semantics is sound and complete for $\mathbf{R}$ (Appendix C).

I don't know of any direct argument, in terms of our intuitive understanding of the truthmaker semantics, which will justify (C1). But there is a kind of bootstrapping argument. First, we note how well summation of states interprets the relevant conditional, as argued in Section 3. This justifies viewing summation itself as an operation which delivers consequent-truthmakers through the combination of conditionaltruthmakers with antecedent-truthmakers. That is, summation behaves as an ontological correlate of modus ponens. Second, where modus ponens goes, so goes modus tollens. An ontic modus tollens will say that a truthmaker for a conditional combined with a consequent-counterexample results in an antecedent-counterexample (or a determinate of one). Now recall (from Section 6) that we analyse conditional counterexamples via matehood. Putting these parts together: combining a conditionaltruthmaker with the mate of some consequent-state yields the mate of some antecedent-state (or one which determines it). That's just what (C4) says.

Now, this argument clearly doesn't show that the truthmaker semantics is committed to (C4). It is intended to show that $(\mathrm{C} 4)$ is a reasonable principle to adopt, by highlighting that there is a justified way of conceptualising summation which supports (C4). I don't know of any philosophical interpretation of a relevant semantics 
which mandates CONTRAPOSITION. A contraposition-supporting semantic feature may be added to the familiar interpretations, but it is not forced by them. The approach suggested here seems to do at least as well.

\section{Excluded Middle}

Our final task in giving a semantics for $\mathbf{R}$ is to secure the validity of excluded middle, $A \vee \neg A$. On the face of it, this is a challenging principle for a truthmaker semantics. For in general, a state need make neither $A$ nor $\neg A$ true. And if an arbitrary state need not pronounce on whether $A$, then it hardly seems that the null state $\square$, which we use to record validity, will do any better.

To guarantee that $A \vee \neg A$ is made true by the null state, we may adopt the following principle:

(6) If $s \leq_{P}$ 口 then $s \preceq s^{M}$

This delivers excluded middle as follows. Suppose $s$ is an arbitrary prime state which determines the null state, $s \leq_{P}$ ․ (Note that we do not assume that the null state must be fully determinate.) Then for any $A$, we can show that either $s \Vdash A$ or $s \Vdash \neg A$. For if $s \Vdash A$ then $s^{M} \Vdash \neg A, s \preceq s^{M}$ (by (6)) and so $s \Vdash \neg A$ (by HEREDITY). So we have either $s \Vdash A$ or $s \Vdash \neg A$ for any prime determinate of a and so, by our analysis of disjunction, $\square \Vdash A \vee \neg A$.

How might we argue for (6)? It turns out that, given our acceptance of the contraposition principle (C4), we can establish (6) on purely logical grounds. First, we establish (7) For any $s \in P, s \preceq s \sqcup s^{M}$ as follows (for $s \in P$ ):

$$
\begin{aligned}
& s^{M} \preceq s^{M} \\
& \Leftrightarrow s^{M} \preceq s^{M} \sqcup s^{M} \\
& \Leftrightarrow s^{M M} \preceq s^{M} \sqcup s^{M M} \\
& \Leftrightarrow s \preceq s \sqcup s^{M} \quad \text { (commutativity of } \sqcup, s^{M M}=s \text { ) }
\end{aligned}
$$

from which we derive (6):

$$
\begin{aligned}
s \leq \square \quad & \Rightarrow \quad s \sqcup s^{M} \leq \square \sqcup s^{M} \\
& \Leftrightarrow \quad s \sqcup s^{M} \leq s^{M} \\
& \Leftrightarrow \quad s \leq s^{M}
\end{aligned}
$$

$(7$, transitivity of $\leq)$

As a result, we guarantee the validity of excluded middle on accepting the contraposition principle $(\mathrm{C} 1)$. We might find additional motivation for this approach, independently of (C1), as follows. The null state is relevant to each $A$, if only in that it truthmakes the trivial implication from $A$ to itself (which many states do not). Any determinate of the null state must therefore remain relevant to each $A$. But if a state relevant to some $A$ is to count as determinate enough (as we characterise the prime states), then it should settle $A$ one way or another. If so, then any prime determinate of the null state must settle each $A$ one way or another, which (as the above reasoning shows) will guarantee the validity of excluded middle. 


\section{Other Logics}

Logics other than $\mathbf{R}$ may be obtained by tweaking various features of the semantics, but these will not always be philosophically acceptable. I'll briefly outline some of the options.

The relevant logic $\mathbf{E}$ may be axiomatised like $\mathbf{R}$ but with RESTRICTED ASSERTION, $((A \rightarrow A) \rightarrow B) \rightarrow B$, in place of PERMUTATION. The semantics must drop the assumption that $\sqcup$ commutes $(s \sqcup u=u \sqcup s)$. RESTRICTED ASSERTION is valid so long as the null state is a right identity, $s \sqcup \square=s$.. A complete truthmaker semantics for $\mathbf{E}$ requires a more radical amendment than merely dropping commutativity, however. We must reformulate the conditions on $\sqcup$ using refinement in place of identity. Thus idempotence becomes $s \preceq s \sqcup s$, right identity $s \leq s \sqcup \square$, and so on. The intuitive reason is that $A$ is equivalent to $(A \rightarrow A) \rightarrow A$ in $\mathbf{R}$, where it encodes ' $A$ is true', but not in $\mathbf{E}$, where it encodes ' $A$ is necessary'. Without this equivalence, our attempts to demonstrate identities $s=t \sqcup u$ in the completeness proof are frustrated.

With conditions on $\sqcup$ formulated in terms of inequalities $s \preceq t \sqcup u$, we can give semantics for a range of weaker logics, including ticket entailment $\mathbf{T}$, using the conditions given in [8]. However, none of these moves will be underpinned by our philosophical understanding of $\sqcup$ in terms of parthood, which demands both commutativity and the identity formulation of the conditions. Genuine truthmaker semantics results in a logic whose implicational fragment is at least as strong as $\mathbf{R} \rightarrow$.

Stronger logics are available, at the expense of relevance. $\mathbf{R M}$ adds to $\mathbf{R}$ the MINGLE axiom, $A \rightarrow(A \rightarrow A)$. The truthmaker semantics for $\mathbf{R M}$ is given by closed models, where a model is closed when $s \sqcup u \Vdash A$ whenever $s \Vdash A$ and $u \Vdash A$, and $s \sqcup u \dashv A$ whenever $s \dashv A$ and $u \dashv A$. (Within a closed model, sets of states correspond to what [11] calls closed propositions.) Philosophically, closure seems highly reasonable: combining two truthmakers for a claim results in a state which both guarantees and is clearly relevant to that claim's truth. However, we cannot guarantee closure in a model merely by imposing it on the valuation functions (for the falsemaking clause for $\rightarrow$ depends on what a related state does not make true, over which the imposition of closure to less complex sentences has no say.)

We might also consider a semantics defined in terms of inexact, rather than exact, truthmaking. (Recall that an inexact truthmaker for $A$ is one which has an exact truthmaker for $A$ as a part.) If we re-define validity in terms of inexact truthmaking at the null state of all models, or all states of all models, we get the same set of valid sentences as previously. For the null state has no parts but itself and so it inexactly truthmakes whatever it exactly truthmakes.

Alternatively, we might consider an entailment $A \models B$ to hold just in case any state in any model inexactly truthmakes $B$ when it inexactly truthmakes $A$. On this approach, o behaves as extensional conjunction, made true wherever its conjuncts are. Taking $\circ$ as conjunction and ignoring $\wedge, A=B$ just in case $A$ tautologically entails $B$ in Anderson and Belnap's [1] sense, as van Fraassen [32] shows. Taking $A \rightarrow B$ to be valid when $A$ tautologically entails $B$ results in first degree entailment, FDE [2].

Finally, we might consider a conditional $\Rightarrow$, defined so that $s \Vdash A \Rightarrow B$ iff, for all $u \Vdash A$ and some $t \sqsubseteq s \sqcup u, t \Vdash B$. (When this is so, $s \sqcup u$ inexactly makes $B$ true.) Then positive paradox $A \rightarrow(B \rightarrow A)$ will be valid, since the sum of an $A$ - 
state and $B$-state will inexactly make $A$ true. The resulting conditional is then at least as strong as intuitionistic implication. In the presence of negation, we have classical logic (again with $\circ$ as conjunction). If we ignore $\neg$ and introduce a constant $\perp$, we can give an intuitionistic semantics in the style of [10], by taking $\perp$ to be inexactly made true by states which inexactly make true every primitive. (We can then define an intuitionistically acceptable negation $\sim A$ as $A \rightarrow \perp$.)

\section{Conclusion}

Truthmaker semantics provides philosophically well-motivated home for relevance logic (for $\mathbf{R}$ at least). Adding a very natural notion of conditionality to standard truthmaker semantics gives a ready-made semantics for $\mathbf{R}^{\rightarrow}$ (Section 3). For the $\neg$-free fragment $\mathbf{R}^{+}$, we need to add the notion of determination, which is justified on philosophical grounds (Section 5). When we add negation, we need to add the notion of matehood to relate truthmakers and falsitymakers (Section 6). Achieving a fully contraposing conditional, and hence a full semantics for $\mathbf{R}$, requires interaction between parthood, determination, and matehood. This is plausible, but not mandatory, from the perspective of truthmaker semantics (Section 7). We saw how the intuitive analysis of validity in terms of being made true by the null state can account for the validity of excluded middle (Section 8). The semantics may be adapted for weaker relevant logics (Section 9) but, philosophically, $\mathbf{R}^{+}$is as weak as the truthmaker approach should go.

Open Access This article is licensed under a Creative Commons Attribution 4.0 International License, which permits use, sharing, adaptation, distribution and reproduction in any medium or format, as long as you give appropriate credit to the original author(s) and the source, provide a link to the Creative Commons licence, and indicate if changes were made. The images or other third party material in this article are included in the article's Creative Commons licence, unless indicated otherwise in a credit line to the material. If material is not included in the article's Creative Commons licence and your intended use is not permitted by statutory regulation or exceeds the permitted use, you will need to obtain permission directly from the copyright holder. To view a copy of this licence, visit http://creativecommons.org/licenses/by/4.0/.

\section{Appendix A: The Logic R}

$\mathbf{R}^{\rightarrow}$ is the implicational fragment of $\mathbf{R}$, axiomatised by:

(IDENTITy) $A \rightarrow A$

(Prefixing) $(A \rightarrow B) \rightarrow((C \rightarrow A) \rightarrow(C \rightarrow B))$

(Contraction) $(A \rightarrow(A \rightarrow B)) \rightarrow(A \rightarrow B)$

(Permutation) $(A \rightarrow(B \rightarrow C)) \rightarrow(B \rightarrow(A \rightarrow C))$

with modus ponens, $A, A \rightarrow B / B$ as the only rule. For $\wedge$ and $\vee$, we add:

$(\wedge$-Introduction $)((A \rightarrow B) \wedge(A \rightarrow C)) \rightarrow(A \rightarrow B \wedge C)$

(^-ELimination) $A \wedge B \rightarrow A \quad A \wedge B \rightarrow B$

( $\vee$-Introduction) $A \rightarrow A \vee B \quad B \rightarrow A \vee B$

$(\vee$-ELIMINATION $)((A \rightarrow C) \wedge(B \rightarrow C)) \rightarrow(A \vee B \rightarrow C)$

(Distribution) $(A \wedge(B \vee C)) \rightarrow((A \wedge B) \vee(A \wedge C))$ 
plus adjunction, $A, B / A \wedge B$, as a rule. This gives the positive fragment, $\mathbf{R}^{+}$. For negation, we add:

(Contraposition) $(A \rightarrow B) \rightarrow(\neg B \rightarrow \neg A)$

(REDUCTIO) $(A \rightarrow \neg A) \rightarrow \neg A$

(Double Negation) $\neg \neg A \rightarrow A$

The resulting logic is $\mathbf{R}$. $A \circ B$ can be defined in $\mathbf{R}$ as $\neg(A \rightarrow \neg B)$. In $\mathbf{R}^{+}$, we may treat it is a primitive and add an axiom: $A \circ B \rightarrow C \leftrightarrow(A \rightarrow(B \rightarrow C))$.

\section{Appendix B: Semantics}

We now define formal models, based on the philosophical principles for which I argued through Sections 3-7. To keep proofs brief, I will treat $\circ$ and $\vee$ as defined connectives, with $A \vee B==_{\mathrm{df}} \neg(\neg A \wedge \neg B)$ and $A \circ B={ }_{\mathrm{df}} \neg(A \rightarrow \neg B)$.

Definition 1 (Models) Models are tuples $\left\langle S, P, \square, \sqsubseteq, \leq, M, V^{+}, V^{-}\right\rangle$, where $S$ and $P \subseteq S$ are sets of states; $\sqsubseteq$ and $\preceq$ are partial orders on $S$; $M$ is an operation on $P$; and $V^{+}$and $V^{-}$are functions from $S$ to sets of primitive sentence letters. Models must satisfy the following conditions (where ' $s \preceq P \quad u$ ' abbreviates ' $s \in P$ and $s \preceq u$ '):

(C1) $\sqsubseteq$ is a partial order with a minimal element $\square$ such that any pair $s, u \in S$ has a 巨-least upper bound, $s \sqcup u \in S$.

(C2) $\preceq$ is a partial order which distributes over $\sqcup$ : if $s \preceq u$ then $\forall t: s \sqcup t \preceq u \sqcup t$.

(C3) If $s \preceq_{P} t \sqcup u$, then there is a $t^{\prime} \preceq_{P} t$ such that $s \preceq_{P} t^{\prime} \sqcup u$.

(C4) If $s \preceq_{P} t \sqcup u$ and $u \in P$ then $u^{\bar{M}} \preceq t \sqcup s^{M}$.

(C5) $p \in V^{+} s$ iff $\forall u \preceq{ }_{P} s: p \in V^{+} u$; and $p \in V^{-} s$ iff $\forall u \preceq_{P} s: p \in V^{+} u$.

(C6) For all $s \in P: s^{M M}=s$; $p \in V^{+} s$ iff $p \notin V^{-} s^{M}$; and $p \in V^{-} s$ iff $p \notin V^{+}{ }^{M}$.

\section{Definition 2 (Truthmaking and falsitymaking relations)}

$$
\begin{aligned}
& s \Vdash p \text { iff } p \in V^{+} s \\
& s \dashv p \text { iff } p \in V^{-} s \\
& s \Vdash \neg A \text { iff } s \dashv A \\
& s \dashv \neg A \text { iff } s \Vdash A \\
& s \Vdash A \wedge B \text { iff } s \Vdash A \& s \Vdash B \\
& s \dashv A \wedge B \text { iff } \forall u \leq_{P} s: u \dashv A \text { or } u \dashv B \\
& s \Vdash A \rightarrow B \text { iff } \forall u \Vdash A: s \sqcup u \Vdash B \\
& s \rightarrow A \rightarrow B \text { iff } \forall t \leq_{P} s \quad \exists u \Vdash A: t^{M} \sqcup u \Vdash B
\end{aligned}
$$


These models synthesise ideas from [8] and [26], and many of the subsequent proofs borrow from theirs. (Given the interrelation of these moving parts, we cannot assume that their results hold without proof.) The formal treatment of $\preceq$ using (C2)(C5) derives from Fine, whereas the formal treatment of negation and mates is due to Routley. Fine analyses negation via $\preceq$, whereas here it is analysed directly in terms of falsitymaking. The philosophical interpretation here differs both from Fine's and Routley's. Neither author mentions truthmakers in those works and [8] understands $\preceq$ in terms of extension of a theory, rather than determinacy of states.

Lemma 1 (Disjunction) Disjunction has the following (derived) clauses:

$$
\begin{aligned}
& s \Vdash A \vee B \quad \text { iff } \forall u \leq_{P} s: u \Vdash A \text { or } u \Vdash B \\
& s \dashv A \vee B \quad \text { iff } s \dashv A \& s \dashv B
\end{aligned}
$$

Proof $s \Vdash A \vee B$ iff $s \Vdash \neg(\neg A \wedge \neg B)$ iff $s \dashv \neg A \wedge \neg B$ iff $\forall u \preceq P \quad s: u \dashv \neg A$ or $u \dashv \neg B$ iff $\forall u \preceq P \quad s: u \Vdash A$ or $u \Vdash B . s \dashv A \vee B$ iff $s \dashv \neg(\neg A \wedge \neg B)$ iff $s \Vdash \neg A \wedge \neg B$ iff $s \Vdash \neg A \& s \Vdash \neg A$ iff $s \dashv A \& s \dashv A$.

\section{Lemma 2 (Heredity)}

(a) $s \Vdash A$ iff for all $u \preceq P s: u \Vdash A$

(b) $s \dashv A$ iff for all $u \leq_{P} s: u \dashv A$

Proof By double induction on $A$. The base cases follow immediately from (C5), so suppose both (a) and (b) hold for all $B$ less complex than $A$ and consider these cases.

$-A:=\neg B$. For (a): $s \Vdash \neg B$ iff $s \dashv B$ iff $\forall u \leq_{P} s: u \dashv B$ (by IH) iff $\forall u \preceq P$ $s: u \Vdash \neg B$. For (b): $s \dashv \neg B$ iff iff $s \Vdash B$ iff $\forall u \preceq_{P} s: u \Vdash B$ (by IH) iff $\forall u \leq_{P} s: u \dashv \neg B$.

$-A:=B \wedge C$. For (a): $s \Vdash B \wedge C$ iff $s \Vdash B$ and $s \Vdash C$ iff $\forall u \preceq P s: u \Vdash B$ and $u \Vdash C$ (by IH) iff $\forall u \preceq_{P} s: u \Vdash B \wedge C$. For (b): $s \dashv B \wedge C$ iff $\forall u \preceq_{P} s: u \dashv B$ or $u \dashv C$ iff $\forall u \preceq P \quad s \forall t \preceq P u: t \dashv B$ or $t \dashv C$ (if by transitivity of $\preceq$; only if by IH) iff $\forall u \leq_{P} s: u \dashv \bar{B} \wedge C$.

- $A:=B \rightarrow C$. For (a) left-to-right: assume $s \Vdash B \rightarrow C, t \Vdash B$, and $u \preceq P s$. Then $s \sqcup t \Vdash C$ and $u \sqcup t \preceq s \sqcup t$ (by C2). Now assume $v \preceq P \quad u \sqcup t$. Then $v \preceq P s \sqcup t$ (by transitivity of $\preceq$ ), so $v \Vdash C$ (by IH only if). Since $v$ was arbitrary, we infer $\forall v \preceq u \sqcup t: C$, hence $u \sqcup t \Vdash C$ (by IH if), and so $u \Vdash B \rightarrow C$.

Right-to-left is by contraposition. Assume $s \Vdash B \rightarrow C$. So for some $t \Vdash B$, $s \sqcup t$ It $C$. By hypothesis, there is some $v \preceq_{P} s \sqcup t$ such that $v \Vdash C$. By (C3), there is some $u \preceq P s$ such that $v \preceq u \sqcup t$. By hypothesis, $u \sqcup t \Vdash H C$ and so $u \Vdash B \rightarrow C$.

For (b), left-to-right: assume $s \dashv B \rightarrow C, u \preceq_{P} s$, and $v \preceq_{P} u$. Then $v \preceq_{P} s$, hence $\exists t \Vdash B: v^{M} \sqcup t \Vdash C$ for any $v \preceq_{P} u$, hence $u \dashv B \rightarrow C$ for any $u \preceq_{P} s$. Right-to-left: assume $\forall u \preceq_{P} s: u \dashv B \rightarrow C$. Then since $u \preceq_{P} u$, by definition $\exists t \Vdash B: u^{M} \sqcup t \Vdash C$ for any $u \preceq P s$ and so $s \dashv B \rightarrow C$. 
Corollary 1 (a) If $s \Vdash A$ and $s \preceq u$ then $u \Vdash A$; and if $s \dashv A$ and $s \preceq u$ then $u \dashv A$.

(b) If $s \in P$, then the $\wedge \dashv 1, \vee \Vdash$, and $\rightarrow \dashv$ clauses simplify to:

$$
\begin{aligned}
& s \dashv A \wedge B \quad \text { iff } s \dashv A \text { or } u \dashv B \\
& s \Vdash A \vee B \quad \text { iff } s \Vdash A \text { or } s \Vdash B \\
& s \dashv A \rightarrow B \quad \text { iff } \exists u \Vdash A: s^{M} \sqcup u \Vdash B
\end{aligned}
$$

(c) $s \Vdash A$ implies $s \Vdash A \vee B$

Proof For (a), assume $s \Vdash A, u \preceq s$, and $t \preceq_{P} u$. By transitivity of $\preceq_{,} t \preceq_{P} s$. So by lemma $2, t \Vdash A$ for any $t \preceq P u$, and hence $u \Vdash A$. The $\dashv$ case is similar. For (b), the left-to-right directions follow by the reflexivity of $\preceq$ (given $s \in P$ ) and the right-toleft directions follow directly from lemma 2. For (c), $s \Vdash A$ iff $\forall u \preceq_{P} A$ (2) only if $\forall u \preceq_{P} A \vee B$ (b) iff $s \Vdash A \vee B(2)$.

Lemma 3 (Crossover) For all prime states $s$ :

(a) $s \Vdash A$ iff $s^{M}$ Al $A$

(b) iff $s^{M} \Vdash A$

Proof By double induction on $A$. Assume $s$ is prime throughout. The base cases follow immediately from (C5), so suppose both (a) and (b) hold for all $B$ less complex than $A$ and consider these cases.

$-A:=\neg B$. For (a): $s \Vdash \neg B$ iff $s \dashv B$ iff $s^{M} \Vdash A$ (by IH) iff $s^{M} A \| \neg A$. (b) is similar.

$-A:=B \wedge C$. For (a): $s \Vdash B \wedge C$ iff $s \Vdash B$ and $s \Vdash C$ iff $s^{M} A \| B$ and $s^{M} A l C$ (by IH) iff $s^{M} A \| B \wedge C$ (1(b)). For (b): $s \dashv B \wedge C$ iff $s \dashv B$ or $s \dashv C$ iff $s^{M} \Vdash B$ or $s^{M} \Vdash C$ (by IH) iff $s^{M} \Vdash B \wedge C$.

$-A:=B \rightarrow C$. For (a): $s \Vdash B \rightarrow C$ iff $\forall u \Vdash B: s \sqcup u \Vdash C$ iff $\neg \exists u \Vdash$ $B: s^{M M} \sqcup u \Vdash C$ (given $s^{M M}=u$ ) iff $s^{M}$ Al $B \rightarrow C$ (1b, given $s \in P$ ). For (b): $s \dashv B \rightarrow C$ iff $\exists u \Vdash A: s^{M} \sqcup u \Vdash B$ (1b) iff $s^{M} \Vdash B \rightarrow C$.

Corollary 2 (Star) For any $s \in P: s \Vdash \neg A$ iff $s^{M} \Vdash A$ and $s \dashv \neg A$ iff $s^{M}$ Al $A$.

Corollary 3 (Negation) (a) $s \Vdash \neg A$ iff $\forall u \leq_{P} s: u^{M} \Vdash A$

(b) $s \dashv \neg A$ iff $\forall u \leq_{P} s: u^{M}$ Al $A$

Corollary 4 (Equivalent Models) Let $\| \vdash$ be the relation defined recursively by the $\Vdash$-clauses for $p, A \wedge B$, and $A \rightarrow B$, and 3(a) for $\neg A$. Then for any model $M: s \| \Vdash A$ iff $s \Vdash A$.

Proof 2 is immediate from definition 1 and crossover (3). 3 follows from 2 and heredity (2). 4 follows from 3. 
2 shows that, for prime states at least, matehood can do the work of the Routley star. 3 shows that we can effectively do away with the falsitymaking relation altogether and work only with truthmaking (as reflected in 4). This fact will simplify the completeness proof.

We can now derive the expected clauses for intensional conjunction from its definition $A \circ B==_{\mathrm{df}} \neg(A \rightarrow \neg B)$ :

Lemma 4 (Intensional conjunction) Intensional conjunction has the following (derived) clauses:

$$
\begin{aligned}
& s \Vdash A \circ B \quad \text { iff } \exists t u: t \Vdash A, u \Vdash B \& s \leq t \sqcup u \\
& s \dashv A \circ B \quad \text { iff } \forall u \Vdash A: s \sqcup u \dashv B
\end{aligned}
$$

Proof For the truthmaking clause, left-to-right: suppose $s \Vdash \neg(A \rightarrow \neg B)$ and let $s^{+} \preceq_{P} s$. Then by definition, $s^{+M} \sqcup t \Vdash \neg B$ for some $t \Vdash A$. By lemma $2, u \Vdash \neg B$ for some $u \preceq_{P} s^{+M} \sqcup t$, hence by $2, u^{M} \Vdash B$ and by (C4), $s^{+} \preceq_{P} t \sqcup u^{M}$. This holds for all $s^{+} \preceq_{P} s$ and so $s \preceq t \sqcup u^{M}, t \Vdash A$, and $u \Vdash B$. Right-to-left: suppose $t \Vdash A, u \Vdash B$, and $s \preceq t \sqcup u$ and assume $s^{+} \preceq P s$. By (C3), $s^{+} \preceq t \sqcup u^{+}$for some $u^{+} \preceq_{P} u$ and so, by (C4), $u^{+M} \preceq_{P} s^{+M} \sqcup t$. By $2, u^{+} \Vdash B$, so by $2, u^{+M} \Vdash \neg B$ and so by $2, s^{+M} \sqcup t \Vdash \neg B$. Since $s^{+}$is prime, by definition $s \dashv A \rightarrow \neg B$ and so $s \Vdash \neg(A \rightarrow \neg B)$.

For the falsitymaking clause: $s \dashv \neg(A \rightarrow \neg B)$ iff $s \Vdash A \rightarrow \neg B$ iff $\forall u \Vdash A$ : $s \sqcup u \Vdash \neg B$ iff $\forall u \Vdash A: s \sqcup u \dashv B$.

As expected, a truthmaker for both conjuncts truthmakes their intensional conjunction, and any determinate of a falsemaker for the intensional conjunction is a falsemaker for at least one of its disjuncts:

Corollary 5 (i) If $s \Vdash A$ and $s \Vdash B$ then $s \Vdash A \circ B$; and (ii) if $s \dashv A \circ B$ then, for any $u \preceq P$, either $u \dashv A$ or $u \dashv B$.

Proof For (i): since $s \preceq s \sqcup s, s \Vdash A \& s \Vdash B$ implies $s \Vdash A \circ B$. For (ii): suppose $s \dashv A \circ B$ and $u \preceq P s$ but $u$ Al (to show $u \dashv B$ ). Then by $2, u \Vdash A \circ B$, by $3, u^{M} \Vdash A$, and so by $4, u \sqcup u^{M} \dashv B$. Since $u^{M} \preceq_{P} u^{M} \sqcup u^{M}$, (C4) implies $u^{M M} \preceq_{P} u^{M M} \sqcup u^{M}$, hence $u \preceq_{P} u \sqcup u^{M}$ and, by $2,2, u \dashv B$.

As is usual in models for relevant logics, we define validity in terms of truth(making) at a designated state $\square$ in all models. Since our models feature both truthmaking and falsitymaking relations, we might define entailment in terms of preservation of truthmakers (from premises to conclusion), or in terms of falsitymaker preservation (from conclusion to premises), or both. As it happens, it doesn't matter which definition we adopt. 
Definition 3 (Validity) $A$ is true on $M, M \Vdash A$, iff $\square \Vdash A$ in $M$. $A$ is valid, $\models A$, iff true on all models. A set $\Gamma$ is true on $M$ when all its members are.

Definition 4 (Entailment) $\Gamma$ entails $A$ on $M, \Gamma \models_{M} A$, iff for all states $s$ in $M$, $s \Vdash A$ if $s \Vdash \Gamma$. $\Gamma$ 2-entails $A$ on $M, \Gamma \models{ }_{M}^{2} A$, iff for all states $s$ in $M$ : (i) $s \Vdash \Gamma$ only if $s \Vdash A$ and (ii) $s \dashv A$ only if $s \dashv \Gamma$. Entailment (and 2-entailment) simpliciter is entailment (2-entailment) on all models.

Lemma 5 (Entailment) (a) $M \models A \rightarrow B$ iff $A=_{M} B$ (b) $A \models_{M}^{2} B$ iff $A \models_{M} B$

Proof For (a): $M \models A \rightarrow B$ iff in $M$, $\square \Vdash A \rightarrow B$ iff for all $s \Vdash A$ in $M: s \sqcup \square \Vdash B$ iff for all $s$ in $M: s \Vdash A$ only if $s \Vdash B$ (since $s \sqcup \square=s$ ) iff $A \models_{M} B$. For (b), left-toright is trivial. For right-to-left, assume $A \models_{M} B$. Then $s \dashv B$ iff $\forall u \preceq_{P} \quad s: u \dashv B$ (lemma 2) iff $u^{M} \Vdash \forall$ (lemma 3) iff $u^{M} \Vdash A$ (since $A \models_{M} B$ ) iff $u \dashv A$ (3) iff $u \dashv A(2)$, and so $A \models_{M}^{2} B$.

Note the following corollary, which holds even in the absence of (C1):

Corollary 6 (Rule Contraposition) $\models A \rightarrow B$ iff $\models \neg B \rightarrow \neg A$.

Proof For any model $M: \models_{M} A \rightarrow B$ iff $A \models_{M} B$ iff $A=_{M}^{2}$ iff $\forall s:(s \dashv B$ only if $s \dashv A)$ iff $\forall s:(s \Vdash \neg B$ only if $s \Vdash \neg A)$ iff $\neg B \models_{M} \neg A$ iff $\models_{M} \neg B \rightarrow \neg A$.

\section{Appendix C: Soundness and Completeness}

Theorem 1 (Soundness) If $A \in \boldsymbol{R}$ then $\models A$

Proof We need to show that each axiom of $\mathbf{R}$ is valid and that both modus ponens and adjunction preserve validity. For modus ponens, if $\square \Vdash A \rightarrow B$ then $s \Vdash A$ implies $\square \sqcup s=s \Vdash B$, hence if $\square \Vdash A$ then $\square \Vdash B$. For adjunction, if $\square \Vdash A$ and $\square \Vdash B$ then $\square \Vdash A \wedge B$.

For the axioms: each is of the form $A \rightarrow B$. So, given lemma 5, we need show only that, for any state $s$ in any model, if $s \Vdash A$ then $s \Vdash B$. IDENTITY, $\wedge$-ELIMINATION, and DOUBLE NEGATION are trivial, and $\vee$-INTRODUCTION is immediate from corollary 1(c). The remaining cases are as follows:

Prefixing: Suppose $s \Vdash A \rightarrow B$ and assume $t \Vdash C \rightarrow A$ and $u \Vdash C$. We show $(s \sqcup t) \sqcup u \Vdash B$. By definition, we have $t \sqcup u \Vdash A$ and so $(t \sqcup u) \sqcup s \Vdash B$. Since $(t \sqcup u) \sqcup s=s \sqcup(t \sqcup u)$, the result follows.

Contraction: Suppose $s \Vdash A \rightarrow(A \rightarrow B)$ and assume $u \Vdash A$, to show $s \sqcup u \Vdash$

$B$. Then $(s \sqcup u) \sqcup u \Vdash B$ and, since $(s \sqcup u) \sqcup u=s \sqcup(u \sqcup u)=s \sqcup u$, the result follows. 
Permutation: Suppose $s \Vdash A \rightarrow(B \rightarrow C)$ and assume $t \Vdash B$ and $y \Vdash A$, to show $(s \sqcup t) \sqcup u \Vdash C$. Then $s \sqcup u \Vdash B \rightarrow C$, so $(s \sqcup u) \sqcup t \Vdash C$. Since $(s \sqcup u) \sqcup t=(s \sqcup t) \sqcup u$, the result follows.

$\wedge$-Introduction: Suppose $s \Vdash(A \rightarrow B) \wedge(A \rightarrow C)$ and assume $u \Vdash A$, to show $s \sqcup u \Vdash B \wedge C$. Then $s \Vdash(A \rightarrow B)$ and $s \Vdash(A \rightarrow C)$, hence $s \sqcup u \Vdash B$ and $s \sqcup u \Vdash C$, and so $s \sqcup u \Vdash B \wedge C$.

$\vee$-Elimination: Assume $s \Vdash(A \rightarrow C) \wedge(B \rightarrow C)$ and $u \Vdash A \vee B$. Then $s \Vdash A \rightarrow C, s \Vdash B \rightarrow C$. Now consider any $t \preceq_{P} s \sqcup u$. By (C1), there is some $v \preceq_{P} u$ such that $t \preceq_{P} s \sqcup v$. So $v \Vdash A \vee B$, hence $v \Vdash A$ or $v \Vdash B$ and, either way, $s \sqcup v \Vdash C$. Then by lemma $2, t \Vdash C$, for all $t \preceq_{P} s \sqcup u$, and hence $s \sqcup u \Vdash C$.

Distribution: Assume $s \Vdash A \wedge(B \vee C)$, so that $s \Vdash A$ and $s \Vdash B \vee C$. Assume $u \in P$ and $u \preceq s$. Then $u \Vdash B$ or $u \Vdash C$; and, given lemma $2, u \Vdash A$. So either $u \Vdash A \wedge B$ or $u \Vdash A \wedge C$, and so $s \Vdash(A \wedge B) \wedge(A \wedge C)$.

Contraposition: Assume $s \Vdash A \rightarrow \neg B$ but $s \Vdash B \rightarrow \neg A$. Then $\exists u \Vdash B$ : $s \sqcup u \Vdash \neg A$. Then $\exists t \preceq P s \sqcup u$ st $t \Vdash \neg A$, so $t^{M} \Vdash A$. So $s \sqcup t^{M} \Vdash \neg B$. By (C1), there is a $v \preceq_{P} u$ such that $t \preceq_{P} s \sqcup v$, so by (C1), $v^{M} \preceq_{P} s \sqcup t^{M}$, so $v^{M} \Vdash \neg B$, so $v \Vdash B$, so $u \Vdash B$. Contradiction.

Reductio: Assume $s \Vdash A \rightarrow \neg A$ but $s \Vdash \neg A$. Then $s^{M} \Vdash A$, so $s \sqcup s^{M} \Vdash \neg A$. We also have $s^{M} \preceq s^{M} \sqcup s^{M}$ (reflexivity and idempotence of $\sqcup$ ), hence $s \preceq s \sqcup s^{M}$ (by $\mathrm{C} 1$ and $s^{\overline{M M}}=s$ ), and so (by 2) $s \Vdash \neg A$ : contradiction.

Definition 5 (Deduction and Theories) $\Gamma \vdash_{\mathbf{R}} A$ just in case there is a finite sequence of sentences $A_{1}, \ldots, A_{n}$ where $A=A_{n}$ and each $A_{i \leq n}$ is either in $\Gamma$, or the conjunction of some previous $A_{j}, A_{k}$ (i.e. $j, k<i$ ), or for some $A_{k<i}$, we have $A_{k} \rightarrow A_{i} \in \mathbf{R}$. A theory $T$ is any set of sentences closed under deduction: if $A \in T$ and $\Gamma \vdash_{\mathbf{R}} A$ then $A \in T$. A theory is prime iff either $A \in T$ or $B \in T$ whenever $A \vee B \in T . T_{\mathbf{R}}$ and $P_{\mathbf{R}}$ are the sets of all theories and all prime theories, respectively.

This definition of deduction is not the classical one, for we must ensure that theorems are not automatically derivable from any premises. We do not have $B \vdash_{\mathbf{R}} A \rightarrow$ $A$, for example, even though $A \rightarrow A$ is valid.

Lemma 6 (Prime theories) Assume that $\Gamma$ is closed under disjunction and $T$ is a theory which does not intersect $\Gamma$. Then there is a prime theory $T^{+} \supseteq T$ which does not intersect $\Gamma$.

Proof A standard syntactic result for theories in R; see, e.g., Fine [8].

Definition 6 (Canonical model) The canonical model $\mathcal{M}^{\mathbf{R}}$ is a tuple

$$
\left\langle T_{\mathbf{R}}, P_{\mathbf{R}}, \mathbf{R}, \sqsubseteq_{\mathbf{R}}, \supseteq, M_{\mathbf{R}}, V_{\mathbf{R}}^{+}, V_{\mathbf{R}}^{-}\right\rangle
$$

where $T_{\mathbf{R}}, P_{\mathbf{R}}$, and $\mathbf{R}$ are as above and:

$-S \sqcup_{\mathbf{R}} U=\{B \mid \exists A \in U: A \rightarrow B \in S\}$; set $S \sqsubseteq_{\mathbf{R}} U$ iff $S \sqcup_{\mathbf{R}} U=U$

- For $S \in P_{\mathbf{R}}, S^{M_{\mathbf{R}}}=\{A \mid \neg A \notin S\}$ 
$-V_{\mathbf{R}}^{+} S=\{p \mid p \in S\}$

$-V_{\mathbf{R}}^{-} S=\{p \mid \neg p \in S\}$

The canonical model deviates from our philosophical characterisation in that (i) $M_{\mathbf{R}}$ here is none other than the standard canonical model Routley star and (ii) we model determination using the converse of set inclusion. Nevertheless, the approach meets the the formal requirements on a model and greatly simplifies the completeness proof.

Lemma 7 (Theory heredity) $A \in S$ iff, for all prime $U \supseteq S, A \in S$.

Proof Left-to-right is trivial. For right-to-left, assume $A \notin S$, and let $\Gamma=\left\{B \mid B \dashv \vdash_{\mathbf{R}} A\right\}$. Then $\Gamma$ is disjunction-closed (since $A \dashv \vdash_{\mathbf{R}} A \vee A$ ) and $\Gamma \cap S=$ $\emptyset$. So by lemma 6 , there is a prime $U \supseteq S$ such that $\Gamma \cap U=\emptyset$, hence $A \notin U$. Contraposing, if $A \in U$ for all $U \supseteq S$ then $A \in S$.

Lemma $8 \mathcal{M}^{\boldsymbol{R}}$ is a model.

Proof We need to demonstrate that each of (C1)-(C6) hold of $\mathcal{M}^{\mathbf{R}}$ :

(C1) We must show that (i) $\sqsubseteq_{\mathbf{R}}$ is a partial order on $T_{\mathbf{R}}$; (ii) $\mathbf{R}$ is its minimal element; and (iii) $S \sqcup_{\mathbf{R}} U \in T_{\mathbf{R}}$ for every $S, U \in \mathbf{R}$.

(i): A standard proof (e.g. Dunn and Restall [7]) shows that the PERMUTATION, PREFIXING, and CONTRACTION axioms guarantee that $\sqcup_{\mathbf{R}}$ is commutative, associative, and idempotent. It is then a standard result of order theory that defining $S \sqsubseteq_{\mathbf{R}} U$ iff $S \sqcup_{\mathbf{R}} U=U$ results in a partial order.

(ii): If $A \in S$ then, since $A \rightarrow A \in \mathbf{R}$, we have $A \in \mathbf{R} \sqcup_{\mathbf{R}} S$. If $A \in \mathbf{R} \sqcup_{\mathbf{R}} S$ then $B \rightarrow A \in \mathbf{R}$ for some $B \in S$. Then $B \vdash_{\mathbf{R}} A$ (definition 5) and so, since $S$ is a theory (definition 5), $A \in S$. So $\mathbf{R} \sqcup_{\mathbf{R}} S=S$ and hence $\mathbf{R} \sqsubseteq \mathbf{R} S$ for every state $S$.

(iii): This requires that each $S \sqcup_{\mathbf{R}} U$ is a theory (by definition 5's lights), which is a standard syntactic result (e.g. Fine [8]).

(C2) Clearly $\supseteq$ is a partial order; we show it distributes over $\sqcup_{\mathbf{R}}$. Suppose $S \supseteq U$ and $A \in U \sqcup T$. Then for some $B \in U, B \rightarrow A \in T$ and $B \in S$, hence $A \in S \sqcup T$. So $S \sqcup T \supseteq U \sqcup T$.

(C3) Suppose $S \supseteq T \sqcup U$ and $S$ is prime, and let $\Gamma=\{A \mid \exists B \notin S: A \rightarrow B \in U\}$. To show $\Gamma$ is closed under disjunction, suppose $A_{1}, A_{2} \in \Gamma$. Then there are $B_{1}, B_{2} \notin S$ such that $A_{1} \rightarrow B_{1}, A_{2} \rightarrow B_{2} \in U$. Since $A_{1} \rightarrow B_{1}, A_{2} \rightarrow B_{2} \vdash_{\mathbf{R}}$ $A_{1} \vee A_{2} \rightarrow B_{1} \vee B_{2}$ and $U$ is $\vdash_{\mathbf{R}}$-closed, we have $A_{1} \vee A_{2} \rightarrow B_{1} \vee B_{2} \in U$. Since $S$ is prime, $B_{1} \vee B_{2} \notin S$, hence $A_{1} \vee A_{2} \in \Gamma$. Moreover, $\Gamma$ does not intersect $T$. For if there were any $A \in \Gamma \cap T$ then, by definition, there would be some $B \notin S$ such that $A \rightarrow B \in U$, hence $B \in S$ : contradiction. So, by lemma 6 , there is a prime theory $T^{+} \supseteq T$ which also does not intersect $\Gamma$. Now suppose $B \in T^{+} \sqcup U$. Then there is an $A \in T^{+}$such that $A \rightarrow B \in U$, hence $B \in S$ (else $\Gamma$ and $T^{+}$would intersect) and so $T^{+} \sqcup U \subseteq S$. 
(C4) Suppose $S, U \in P, S \supseteq T \sqcup U$ and $A \in T \sqcup S^{M}$. Then for some $B \in S^{M}$, $B \rightarrow A \in T$ and hence, given Contraposition, $\neg A \rightarrow \neg B \in T . B \in S^{M}$ implies $\neg B \notin S$, hence $\neg B \notin T \sqcup U$. Then $\neg A \notin U$ (else we get $\neg B \in S$ ), hence $A \in U^{M}$, and so $U^{M} \supseteq T \sqcup S^{M}$.

(C5) Trivial, given lemma 7.

(C6) We show that, for all $s \in P$ : (i) $S^{M M}=S$; (ii) $p \in V^{+} s$ iff $p \notin V^{-} s^{M}$; and (iii) $p \in V^{-} s$ iff $p \notin V^{+} s^{M}$. We use the fact that $A \dashv \vdash_{\mathbf{R}} \neg \neg A$ and so, since $S$ is a theory, $\neg \neg A \in S$ iff $A \in S$. For (i): $A \in S^{M_{\mathbf{R}} M_{\mathbf{R}}}$ iff $\neg A \notin S^{M_{\mathbf{R}}}$ iff $\neg \neg A \in S$ iff $A \in S$. For (ii): $p \in V_{\mathbf{R}}^{+} S$ iff $p \in S$ iff $\neg \neg p \in S$ iff $\neg p \notin S^{M_{\mathbf{R}}}$ iff $p \in V_{\mathbf{R}}^{-} S^{M_{\mathbf{R}}}$. For (iii): $p \in V_{\mathbf{R}}^{-} S$ iff $\neg p \in S$ iff $p \notin S^{M_{\mathbf{R}}}$ iff $p \notin V_{\mathbf{R}}^{+} S^{M_{\mathbf{R}}}$.

Lemma 9 (Truth) For any state $S$ in $\mathcal{M}^{\boldsymbol{R}}: S \Vdash A$ iff $A \in S$.

Proof By induction on $A$. The base case is given by the definition of $V_{\mathbf{R}}^{+}$; so assume the result holds for all $B$ less complex than $A$ and consider these cases:

$-A:=\neg B . S \Vdash \neg B$ iff $U \Vdash \neg B$ for all prime $U \supseteq S$ (lemma 2) iff $U^{M_{\mathbf{R}}} \Vdash B$ for all prime $U \supseteq S$ (corollary 2) iff $B \notin U^{\bar{M}}$ for all prime $U \supseteq S$ (by hypothesis) iff $\neg B \in U$ for all prime $U \supseteq S$ (definition of $M_{\mathbf{R}}$ ) iff $\neg B \in S$ (lemma 6).

$-A:=B \wedge C . S \Vdash B \wedge C$ iff $S \Vdash B$ and $S \Vdash C$ iff $B \in S$ and $C \in S$ (by hypothesis) iff $B \wedge C \in S$ (since $B, C \vdash_{\mathbf{R}} B \wedge C$ ).

$-A:=B \rightarrow C$. $S \Vdash B \rightarrow C$ iff $\forall U \Vdash B: S \sqcup_{\mathbf{R}} U \Vdash C$ iff $\forall U \ni B: C \in S \sqcup_{\mathbf{R}} U$ (by hypothesis) iff $B \rightarrow C \in S$ (by definition of $\sqcup_{\mathbf{R}}$ ).

Theorem 2 (Completeness) If $\models A$ then $A \in \boldsymbol{R}$.

Proof By contraposition. Assume $A \notin \mathbf{R}$. Then by lemma $9, \mathbf{R} \Vdash A$ in $\mathcal{M}^{\mathbf{R}}$. and, by lemma $8, \not \neq A$.

Definition 7 (Closed models) A model is closed when, for any of its states $s, u$ : $s \sqcup u \Vdash A$ if $s \Vdash A$ and $u \Vdash A$. $A$ is valid on the class of closed models, $\models_{C} A$, when $\square \Vdash$ in every closed model.

Theorem $3 \boldsymbol{R M}$ is sound and complete on the class of closed models: $A \in \boldsymbol{R M}$ iff $\models C A$.

Proof For soundness: suppose $s, u \Vdash A$ in a closed model $\mathcal{M}$. By closure, $s \sqcup u \Vdash A$, hence $s \Vdash A \rightarrow A$ and so ${ }^{\square} \Vdash A \rightarrow(A \rightarrow A)$. For completeness, we show that the canonical model formed from $\mathbf{R M}$ in place of $\mathbf{R}$ is closed. Suppose $S$ and $U$ are RM-theories, each containing $A$. Since $A \rightarrow(A \rightarrow A)$ is a theorem, $A \rightarrow$ $A \in S$. By construction, $S \sqcup_{\mathbf{R M}} U=\{B \mid \exists A \in U: A \rightarrow B \in S\}$ and so $A \in S \sqcup_{\mathbf{R M}} U$. 


\section{References}

1. Anderson, A., \& Belnap, N. (1962). Tautological entailments. Philosophical Studies, 13(1), 9-24.

2. Anderson, A., \& Belnap, N. (1963). First degree entailments. Mathematische Annalen, 149(4), 302319 .

3. Armstrong, D. (1997). A world of states of affairs. Cambridge: Cambridge University Press.

4. Berto, F., \& Restall, G. (2018). Negation on the australian plan. Journal of Philosophical Logic.

5. Church, A. (1951). The weak positive implication calculus. Journal of Symbolic Logic, 16, 238.

6. Dunn, J. (1966). The algebra of intentional logics. PhD thesis, University of Pittsburgh.

7. Dunn, J., \& Restall, G. (2002). Relevance logic. In Gabbay, D., \& Guenthner, F. (Eds.) Handbook of philosophical logic. 2nd edn., (Vol. 6 pp. 1-136). Dordrecht: Kluwer Academic.

8. Fine, K. (1974). Models for entailment. Journal of Philosophical Logic, 3(4), 347-372.

9. Fine, K. (1976). Completeness for the semilattice semantics with disjunction and conjunction (abstract). Journal of Symbolic Logic, 41(2), 560-560.

10. Fine, K. (2014). Truth-maker semantics for intuitionistic logic. Journal of Philosophical Logic, 43(23), 549-577.

11. Fine, K. (2017). A theory of truth-conditional content I: conjunction, disjunction and negation. Journal of Philosophical Logic, 46(6), 625-674.

12. Fine, K. (2017). A theory of truth-conditional content II: subject-matter, common content, remainder and ground. Journal of Philosophical Logic, 46(6), 675-702.

13. Fine, K. (2017). Truthmaker semantics. In Hale, B., Wright, C., Miller, A. (Eds.) A companion to the philosophy of language (pp. 556-577): Blackwell.

14. Fine, K., \& Jago, M. (2019). Logic for exact entailment. Review of Symbolic Logic, 12(3), 536-56.

15. Forrest, P. (2002). Nonclassical mereology and its application to sets. Notre Dame Journal of Formal Logic, 43(2), 79-94.

16. Mares, E. (2004). Relevant logic: a philosophical interpretation. Cambridge: Cambridge University Press.

17. Mares, E. (2009). Relevance and conjunction. Journal of Logic and Computation. https://doi.org/10. 1093/logcom/exp068.

18. Mares, E.D. (1996). Relevant logic and the theory of information. Synthese, 109(3), 345-360.

19. Moh, S.-K. (1950). The deduction theorems and two new logical systems. Methodos, 2, 56-75.

20. Orlov, I.E. (1928). The calculus of compatibility of propositions. Matematicheskii Sbornik, 35, 263286.

21. Prior, A.N. (1949). Determinables, determinates and determinants. Mind, 58(229), 1-20.

22. Restall, G. (1995). Information flow and relevant logics. In Seligman, J., \& Westerståhl, D. (Eds.) Logic, language and computation: the 1994 Moraga proceedings (pp. 463-477): CSLI Press.

23. Restall, G. (1999). Negation in relevant logics: how I stopped worrying and learned to love the routley star. In Gabbay, D., \& Wansing, H. (Eds.) What is negation? Vol. 13 of applied logic series (pp. 5376): Kluwer.

24. Restall, G. (2004). Relevant and substructural logics. Handbook of the history of logic: logic and the modalities in the twentieth century (pp. 289-398). North-Holland.

25. Rodriguez-Pereyra, G. (2006). Truthmaking, entailment, and the conjunction thesis. Mind, 115(460), 957-982.

26. Routley, R. (1984). The american plan completed: alternative classical-style semantics, without stars, for relevant and paraconsistent logics. Studia Logica, 43(1), 131-158.

27. Routley, R., \& Meyer, R. (1972). The semantics of entailment II. Journal of Philosophical Logic, 1, 53-73.

28. Routley, R., \& Meyer, R. (1972). The semantics of entailment III. Journal of philosophical logic, 1, 192-208.

29. Routley, R., \& Meyer, R. (1973). The semantics of entailment I. In Leblanc, H. (Ed.) Truth, syntax, and semantics (pp. 194-243). North-Holland.

30. Urquhart, A. (1972). Semantics for relevant logics. Journal of Symbolic Logic, 37(1), 159-169.

31. Urquhart, A. (1972). The semantics of entailment. PhD thesis: University of Pittsburgh.

32. van Fraassen, B. (1969). Facts and tautological entailments. Journal of Philosophy, 66(15), 477-487.

33. Wilson, J. (2017). Determinables and determinates. In Zalta, E. (Ed.) The Stanford Encyclopedia of Philosophy. https://plato.stanford.edu/entries/determinate-determinables/.

Publisher's Note Springer Nature remains neutral with regard to jurisdictional claims in published maps and institutional affiliations. 